\title{
Fertility-sparing management and obstetric outcomes in a 20-year-old patient with a Sertoli-Leydig cell tumor of the ovary: A case report and review of the literature
}

\author{
THOMAS STAVRAKIS ${ }^{1}$, IOANNIS KALOGIANNIDIS ${ }^{1}$, STAMATIOS PETOUSIS $^{1}$, \\ CHRISOULA TSOMPANIDOU ${ }^{2}$, DIMITRIS DELKOS ${ }^{1}$, NIKOLAOS PRAPAS $^{1}$ and DAVID ROUSSO ${ }^{1}$ \\ ${ }^{1}$ Third Department of Obstetrics and Gynecology, Aristotle University of Thessaloniki, 54642 Thessaloniki; \\ ${ }^{2}$ Department of Pathology, 'Agios Dimitrios' General Hospital, 54634 Thessaloniki, Greece
}

Received November 24, 2014; Accepted November 26, 2015

DOI: $10.3892 /$ ol.2016.4695

\begin{abstract}
Sertoli-Leydig cell tumors (SLCTs) are an uncommon subtype of sex-cord stromal tumors of the ovary, which most commonly arise in women of reproductive age, creating an issue with regard to the preservation of fertility. The clinical manifestation of SLCTs varies widely, ranging from an asymptomatic clinical profile to extreme virilization. Correct diagnosis of SLCT is crucial and is primarily based on histopathological results. The current study presents the case of a 20-year-old woman who underwent unilateral salpingo-oophorectomy and adjuvant chemotherapy due to the diagnosis of an SLCT of the left ovary. Almost 2 years after the initial surgery, during the follow-up period, the patient conceived normally. Pregnancy was uneventful and the patient vaginally delivered a healthy infant at 38 weeks of gestation. A total of 1 year after delivery (3 years after the initial diagnosis), follow-up of the patient did not reveal any disease recurrence. In conclusion, SLCTs may be adequately treated by fertility-sparing surgery and chemotherapy in young women who wish to preserve their fertility. Natural conception, an uncomplicated pregnancy and a vaginal delivery are possible.
\end{abstract}

\section{Introduction}

Sertoli-Leydig cell tumors (SLCTs) are an uncommon subtype of sex-cord stromal tumors of the ovary. The tumors account for $<0.5 \%$ of all ovarian tumors and are most frequently

Correspondence to: Dr Thomas Stavrakis, Third Department of Obstetrics and Gynecology, Aristotle University of Thessaloniki, 49 Konstantinoupoleos, 54642 Thessaloniki, Greece

E-mail: tstavrakis@gmail.com

Abbreviations: SLCTs, Sertoli-Leydig cell tumors; CT, computed tomography

Key words: Sertoli-Leydig cell tumor, fertility-sparing surgery, sex-cord stromal tumor, virilization, hyperandrogonemia located in one ovary $(1,2)$. SLCTs may produce androgens or estrogens, and their prognosis is associated with tumor differentiation and disease stage (1).

SLCTs are most commonly diagnosed in young women, creating an issue with regard to the preservation of fertility (3). Oncologists should consider the risk of infertility in women of reproductive age who undergo treatment for SLCTs. Patients must be thoroughly informed about the conservative treatment alternatives for genital tract preservation, as well as the potential risk of recurrent disease. The conservative management consists of unilateral salpingo-oophorectomy, usually accompanied by biopsy of the contralateral ovary. Hysterectomy and bilateral salpingo-oophorectomy comprise the radical treatment strategies (4).

The current study presents the case of young female patient with a Sertoli-Leydig ovarian tumor, who underwent conservative management. The obstetric outcome following the conservative treatment approach is presented. A review of the relevant literature is additionally conducted.

\section{Case report}

A 20-year-old woman was admitted to the 'Agios Dimitrios' General Hospital of Thessaloniki (Thessaloniki, Greece) in March 2013 for gynecological investigation due to abdominal pain. The patient mentioned no other symptoms or pathologies. The obstetric history consisted of one vaginal delivery of a live infant. Diagnostic exploration by intravaginal ultrasonography revealed a pelvic mass with a maximum diameter of $13 \mathrm{~cm}$, which originated from the left ovary. These findings were additionally confirmed by computed tomography (CT), and no other pelvic or abdominal features were observed. Tumor serum markers, including cancer antigen (CA)125, CA19-9, carcinoembryonic antigen and $\alpha$-fetoprotein, were all negative.

The patient underwent laparotomic exploration, and gross inspection certified the presence of a cystic tumor of the left ovary with solid components. Peritoneal liquid was sampled for cytological investigation, and a salpingo-oophorectomy of the pathological adnexal mass was performed. Palpation of the retroperitoneal space was negative, while the contralateral adnexa were of normal appearance and size. 
Histopathological examination was performed postoperatively. Macroscopically, the tumor consisted of a well-circumscribed, non-encapsulated, ovoid, solid, cystic tumor of the left ovary measuring $13 \times 8 \times 5.5 \mathrm{~cm}$. Microscopically, the tumor was composed of nodules of primitive gonadal stroma and exhibited compact nesting, irregular branching rows and tubules or cysts of sertoli-like cells separated by fibrous, edematous and hyalinized stroma with few Leydig-like cells. The tumor cells were pleomorphic with hyperchromatic nuclei and numerous mitoses (20 per 10 high power fields). Immunohistochemical staining showed that the tumor cells were positive for cytokeratin AE1/AE3, vimentin and $\alpha$-inhibin. Based on these features, the diagnosis of a poorly-differentiated SLCT (grade 3) without heterogeneous elements (Figs. 1-3) was established. SLCTs are divided into three subtypes: Well, moderately and poorly differentiated SLCTs. The latter two subtypes may contain heterologous elements, a retiform component, or both $(5,6)$.

Three courses of chemotherapy with bleomycin $(30 \mathrm{mg} / \mathrm{dl}$ on days 1,8 and 15$)$, etoposide $\left(100 \mathrm{mg} / \mathrm{m}^{2}\right.$ on days $\left.1-5\right)$ and cisplatin $\left(20 \mathrm{mg} / \mathrm{m}^{2}\right.$ on days $\left.1-5\right)$ were administered to the patient every 21 days following surgery. The tolerance of the patient to the chemotherapy treatment was satisfactory, and only minor side effects, such as tiredness, loss of appetite and temporary hair loss, were observed. Following chemotherapy, the patient attended follow-up appointments every 3 months, consisting of gynecological clinical examination, ultrasonography of the pelvis, tumor marker testing and CT imaging of the abdomen and thorax. At 9 months after the primary surgical treatment, a suspicious mass was detected in the patient's left thorax. A pulmonary biopsy was performed (via a left thoracotomy), which revealed fibrotic tissue and no metastatic disease.

A total of 23 months after the diagnosis of SLCT, the patient achieved a normal conception. The pregnancy was uneventful, with no indications of premature labor. At the 38th week of gestation, the patient vaginally delivered a healthy infant with a birth weight of 3,750 g. Follow-up of the SLCT was continued based on pelvic ultrasonography and tumor markers during pregnancy. CT imaging of the abdomen and thorax was performed prior to conception (during the follow-up period) and again at the end of the puerperium period. A total of 36 months after the initial diagnosis, the patient remains under follow-up and demonstrates no evidence of recurrent disease. Written informed consent was obtained from the patient.

\section{Discussion}

The disease management strategy for the current case was designed based on the patient's reproductive age. Fertility-sparing surgery and adjuvant chemotherapy was the selected treatment approach. Following treatment, a natural conception with an uncomplicated pregnancy was achieved during the patient's follow-up period.

SLCTs are a type of sex-cord stromal tumor of the ovary and may occur at any age; however, they are most frequently observed in younger women, creating an issue with regard to the preservation of fertility (3). Arrhenoblastoma or androblastoma are terminologies that have been previously used to describe SLCTs (1). It has been observed that certain SLCTs

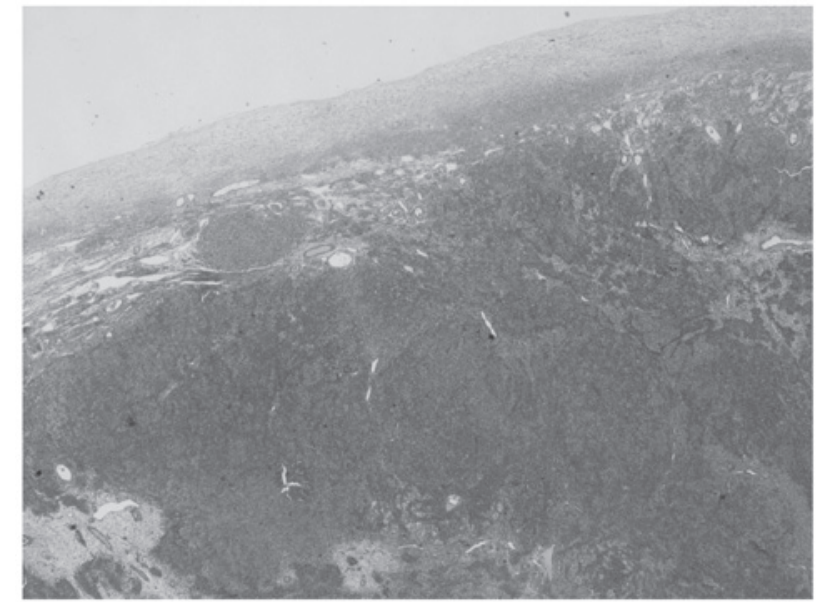

Figure 1. Histopathological image of the ovary with the Sertoli-Leydig tumor. Hematoxylin and eosin staining; magnification, x20.

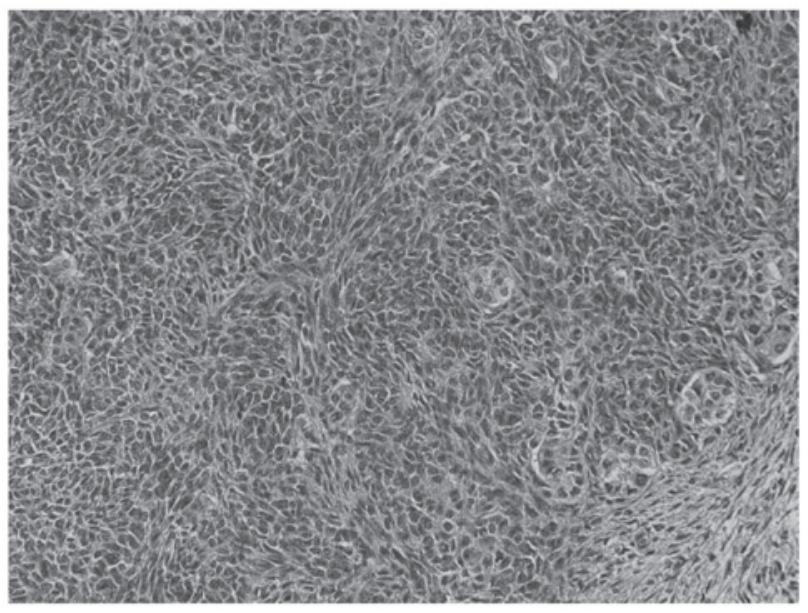

Figure 2. Nodule of spindle-shaped cells and tubular pattern of the Sertoli-Leydig tumor. Hematoxylin and eosin staining; magnification, x200.

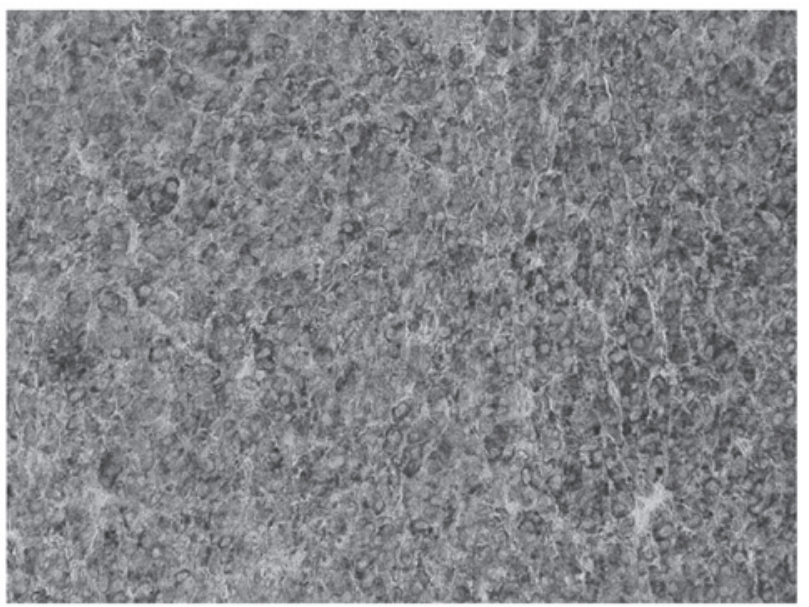

Figure 3. Imunohistochemical staining of the Sertoli-Leydig tumor, positive for $\alpha$-inhibin expression. Magnification, x200.

are associated with mutations in the dicer 1, ribonuclease type III gene $(7,8)$. Patients presenting with this type of SLCT frequently experience a multinodular thyroid goiter 
or alternative uncommon tumors, including Wilms' tumors, cervical rhabdomyosarcoma and pleuropulmonary blastoma, which appear to be hereditary and may be observed in the family history $(7,8)$.

SLCTs of the ovary are classified into 3 grades according to their level of differentiation: Grade 1, well-differentiated; grade 2, moderately-differentiated; and grade 3, poorly-differentiated (9). SLCTs have been reported to most commonly occur unilaterally $(1,10,11)$. The tumor may present as solid, cystic or a combination of the two (1). In the present case, histopathological examination revealed a poorly-differentiated grade 3 tumor.

The diagnosis of an SLCT of the ovary may require considerable time and effort. SLCTs may consist of testicular elements and produce androgens or estrogens (1). Due to androgen secretion, certain SLCT patients may present with masculinization, with symptoms that include anovulation, amenorrhea, defeminization, hirsutism, cliteromegaly, laryngeal protuberance and voice raucity (10). The presence of an ovarian tumor with clinical signs of hormonal disturbances, particularly in younger women, should alert clinicians to the potential presence of an ovarian SLCT. According to a study by Chang et al (12), serum testosterone levels of $>200 \mathrm{ng} / \mathrm{dl}$ primarily indicate the presence of an androgen-producing tumor, commonly confined to the adrenal gland or ovary. The time interval between the oversupply of androgen secretion and the diagnosis of SLCT typically varies from 6-9 months (1). However, as in the present case, in 1/3 of cases, the patient does not exhibit a hormonal disorder (13). Precise diagnosis is achieved via histopathological examination. When handling cases of patients exhibiting secondary amenorrhea with or without virilization, serum androgen levels should be measured during diagnostic procedures, as in certain cases, elevated serum androgen levels may be the only pre-operative indication of an SLCT. A differential diagnosis for alternative causes of hyperandrogenemia (Cushing's syndrome, pituitary tumors or medicine causing androgen hypersecretion) should be performed (14). In addition, less frequently observed estrogenic manifestations (for example, hemorrhage) may be present, particularly in postmenopausal women, and diagnostic curettage typically reveals an irregular proliferative endometrium, hyperplasia or endometrial carcinoma (3).

In certain cases the tumor may be accidentally found during a routine gynecological ultrasound, when suspicious abdominal masses may be observed. If the mass is large it may cause abdominal pain and distension. In addition, rupture of the mass with acute abdominal pain is possible (10). CT and magnetic resonance imaging have made a poor contribution to the diagnosis of SLCTs; $20 \%$ of SLCTs are small in size, and thus unidentifiable, rendering their accurate diagnosis and localization challenging (10). Furthermore, SLCTs are able to coexist with alternative types of ovarian tumors, resulting in patients presenting with mixed symptoms, making diagnosis more challenging. Previous studies have demonstrated that SLCT is associated with mucinous adenocarcinoma and a Brenner tumor of the ovary $(15,16)$.

In the present case, the patient was 20 years old at the time of initial diagnosis. SLCT may be diagnosed at any age, however, is most frequently observed in younger women of reproductive age (3). In a clinicopathological analysis of 207 cases, the average patient age was 34.5 years (1). In a similar study of 40 cases, the median patient age was 28 years (10). In a retrospective study of 21 patients conducted by Sigismondi et al (17), the median patient age was 37 years (range, 16-76 years).

Well-differentiated SLCTs typically present with benign behavior (10). Malignancy is observed in $11 \%$ of moderately-differentiated SLCTs (10-year survival rate, $87 \%$ ) and $59 \%$ of poorly-differentiated SLCTs (10-year survival rate, $41 \%$ ). Furthermore, the 5-year overall survival rate in cases of stage I disease is almost $92 \%$ (17). The primary treatment for SLCTs is surgery, based on the patient age, disease stage and tumor grade $(6,9)$. However, a significant issue arising from disease occurrence in patients of reproductive age is the preservation of fertility (afforded by a cystectomy/adnexectomy with preservation of the rest of the female genital tract). Hysterectomy with bilateral salpingo-oophorectomy, as well as staging surgery (cytology, omentectomy, pelvic-paraaortic lymphadenectomy or peritoneal biopsies), is the optimal approach for patients beyond childbearing age, although the necessity of a lymphadenectomy remains controversial (17). Brown et al (18) demonstrated that in SLCTs, lymph node metastasis is markedly reduced, and sampling of retroperitoneal nodes may be omitted. However, for young women, fertility-sparing surgery (with or without staging surgery) is required, particularly for patients exhibiting stage I disease $(1,17)$. Chemotherapy may be administered following surgical treatment, particularly for tumors demonstrating poor or moderate differentiation (10). According to Gui et al (10), adjuvant chemotherapy does not provide any significant advantages, and it should be considered primarily in cases of poorly- and moderately-differentiated tumors or disease recurrence. Additionally, a previous study (MITO) was able to demonstrate the benefit of the administration of post-operative chemotherapy for patients exhibiting advanced stage (II-IV) disease (17).

In conclusion, SLCTs of the ovary present with a wide range of biological and histopathological features. Precise treatment is based on patient age, tumor differentiation and disease stage. Overall the prognosis of SLCTs is good, particularly in stage I cases, although recurrence may be observed, most commonly in patients exhibiting tumors with moderate or poor differentiation. However, in younger patients conservative surgery for the preservation of fertility is an acceptable approach, which may lead to successful and uneventful conception and pregnancy, as in the present case. Close follow-up must be undertaken in order to monitor the risk of disease recurrence.

\section{References}

1. Young RH and Scully RE: Ovarian Sertoli-Leydig cell tumors. A clinicopathological analysis of 207 cases. Am J Surg Pathol 9: 543-569, 1985.

2. Tomlinson MW, Treadwell MC and Deppe G: Platinum based chemotherapy to treat recurrent Sertoli-Leydig cell ovarian carcinoma during pregnancy. Eur J Gynaecol Oncol 18: 44-46, 1997.

3. Sachdeva P, Arora R, Dubey C, Sukhija A, Daga M and Singh DK: Sertoli-Leydig cell tumor: A rare ovarian neoplasm. Case report and review of literature. Gynecol Endocrinol 24: 230-234, 2008. 
4. Litta P, Saccardi C, Conte L, Codroma A, Angioni S and Mioni R: Sertoli-Leydig cell tumors: Current status of surgical management: Literature review and proposal of treatment. Gynecol Endocrinol 29: 412-417, 2013.

5. Young R: Sex cord-stromal, steroid cell, and other ovarian tumors with endocrine, paraendocrine, and paraneoplastic manifestations. In: Blaustein's Pathology of the Female Genital Tract. Kurman RJ, Hedrick Ellenson L, Ronnett BM (eds). 6th Edition. Springer-Verlag, New York, NY, p815, 2011.

6. National Comprehensive Cancer Network (NCCN): NCCN Clinical Practice Guidelines in Oncology: Ovarian Cancer. 2013. https://www.nccn.org/professionals/physician_gls/f_guidelines. asp. Accessed November 24, 2014.

7. Rio Frio T, Bahubeshi A, Kanellopoulou C, Hamel N, Niedziela M, Sabbaghian N, Pouchet C, Gilbert L, O'Brien PK, Serfas K, et al: DICER1 mutations in familial multinodular goiter with and without ovarian Sertoli-Leydig cell tumors. JAMA 305: 68-77, 2011.

8. Slade I, Bacchelli C, Davies H, Murray A, Abbaszadeh F, Hanks S, Barfoot R, Burke A, Chisholm J, Hewitt M, et al: DICER1 syndrome: Clarifying the diagnosis, clinical features and management implications of a pleiotropic tumour predisposition syndrome. J Med Genet 48: 273-278, 2011

9. Meyer R: The pathology of some special ovarian tumors and their relation to sex characteristics. Am J Obstet Gynecol 22: 697-713, 1931.

10. Gui T, Cao D, Shen K, Yang J, Zhang Y, Yu Q, Wan X, Xiang Y, Xiao Y and Guo L: A clinicopathological analysis of 40 cases of ovarian Sertoli-Leydig cell tumors. Gynecol Oncol 127: 384-389, 2012.

11. Novak ER and Long JH: Arrhenoblastoma of the ovary. Am J Obstet Gynecol 92: 1082-1093, 1965.
12. Chang PL, Lindheim SR, Lowre C, Ferin M, Gonzalez F, Berglund L, Carmina E, Sauer MV and Lobo RA: Normal ovulatory women with polycystic ovaries have hyperandrogenic pituitary-ovarian responses to gonadotropin-releasing hormone-agonist testing. J Clin Endocrinol Metab 85: 995-1000, 2000.

13. Lenhard M, Kuemper C, Ditsch N, Diebold J, Stieber P, Friese K and Burges A: Use of novel serum markers in clinical follow-up of Sertoli-Leydig cell tumours. Clin Chem Lab Med 45: 657-661, 2007.

14. Fleckenstein G, Sattler B, Hinney B, Wuttke W, Osmers R and Emons G: Androblastoma of the ovary: Clinical, diagnostic and histopathologic features. Onkologie 24: 286-291, 2001 (In German).

15. Virk R and Lu D: Mucinous adenocarcinoma as heterologous element in intermediately differentiated Sertoli-Leydig cell tumor of the ovary. Pathol Res Pract 206: 489-492, 2010.

16. Persechini ML, Motton S, Leguevaque $\mathrm{P}$, Donadille F, Escourrou G, Vierasu B, Hamdi S, Bennet A and Caron P: Virilising ovarian tumour: A case associating a Sertoli-Leydig cell tumour and a Brenner tumour. Gynecol Endocrinol 27: 345-350, 2011

17. Sigismondi C, Gadducci A, Lorusso D, Candiani M, Breda E, Raspagliesi F, Cormio G, Marinaccio M and Mangili G: Ovarian Sertoli-Leydig cell tumors. A retrospective MITO study. Gynecol Oncol 125: 673-676, 2012.

18. Brown J, Sood AK, Deavers MT, Milojevic Land Gershenson DM: Patterns of metastasis in sex cord-stromal tumors of the ovary: Can routine staging lymphadenectomy be omitted? Gynecol Oncol 113: 86-90, 2009. 